\title{
Acute effects of power and resistance exercises on hemodynamic measurements of older women
}

\author{
This article was published in the following Dove Press journal: \\ Clinical Interventions in Aging \\ II July 2017 \\ Number of times this article has been viewed
}

Hélio José Coelho-Júnior ${ }^{1,2}$ Maria-Cláudia Irigoyen ${ }^{3}$

Samuel da Silva Aguiar ${ }^{2,4}$

Ivan de Oliveira

Gonçalves ${ }^{2,5}$

Niels Olsen Saraiva

Câmara ${ }^{6}$

Marco Antonio Cenedeze ${ }^{7}$

Ricardo Yukio Asano ${ }^{2,8}$

Bruno Rodrigues'

Marco Carlos Uchida'

'Applied Kinesiology LaboratoryLCA, School of Physical Education, University of Campinas, Barão Geraldo, Campinas, São Paulo, ${ }^{2}$ Center of Health Sciences, University of Mogi das Cruzes, Mogi das Cruzes, ${ }^{3}$ Hypertension Unit, Heart Institute (InCor), Medical School of University of São Paulo, ${ }^{4}$ School of Physical Education, Catholic University of Brasília, Águas Claras, Brasília, ${ }^{5}$ Community Center for Older People of Poá, Poá, ${ }^{6}$ Department of Immunobiology, Laboratory of Transplantation Immunology, Institute of Biomedical Sciences, University of São Paulo, ${ }^{7}$ Nephrology Division, Federal University of São Paulo, ${ }^{8}$ School of Arts, Sciences and Humanities, University of São Paulo, São Paulo, Brazil

Correspondence: Hélio José CoelhoJúnior

Applied Kinesiology Laboratory-LCA, School of Physical Education, University of Campinas-Avenida Érico Veríssimo, 70I-Cidade Universitária "Zeferino Vaz", Barão Geraldo, CEP |3.083-85I, Campinas, São Paulo, Brazil

Tel +55 I| 296। 5143

Email coelhojunior@hotmail.com.br
Purpose: The purpose of this study was to compare the acute effects of resistance training (RT) and power training (PT) on the hemodynamic parameters and nitric oxide (NO) bioavailability of older women.

Materials and methods: A randomized experimental design was used in this study. Twentyone older women (age: $67.1 \pm 4.6$ years; body mass index: $28.03 \pm 4.9 \mathrm{~kg} / \mathrm{m}^{2}$; systolic blood pressure: $135.1 \pm 21.1 \mathrm{mmHg}$ ) were recruited to participate in this study. Volunteers were randomly allocated into PT, RT, and control session (CS) groups. The PT and RT groups underwent a single session of physical exercise equalized by training volume, characterized by 3 sets of $8-10$ repetitions in 8 different exercises. However, RT group performed exercise at a higher intensity (difficult) than PT (moderate) group. On the other hand, concentric contractions were faster in PT group than in RT group. Hemodynamic parameters and saliva samples (for NO quantification) were collected before and during an hour after exercise completion.

Results: Results demonstrated post-exercise hypotension during 35 minutes in the PT when compared to rest period $(P=0.001)$. In turn, RT showed decreased heart rate and double product $(P<0.001)$ during the whole evaluation period after exercise completion compared with the rest period. NO levels increased in the PT and RT during the whole evaluation period in relation to rest period. However, there were no differences between PT, RT, and CS regarding hemodynamic and NO evaluations.

Conclusion: Data indicate that an acute session of power and resistance exercise can be effective to cause beneficial changes on hemodynamic parameters and NO levels in older women.

Keywords: post-exercise hypotension, resistance training, power training, nitric oxide, older people

\section{Introduction}

The aging process is frequently associated with increased prevalence and incidence of chronical degenerative diseases, such as sarcopenia. This progressive disease is mainly characterized by a state of decreased skeletal muscle mass, associated with an impaired capacity to generate strength and power. ${ }^{1-4}$ Sarcopenic patients commonly show impaired capacity to perform the activities of daily living, low glucose metabolism, and vascular alterations (increase in pulse wave velocity), resulting in a poor prognosis in these individuals. ${ }^{1-4}$

Recent guidelines from the American College of Sports and Medicine (ACSM) focused on older people and adults indicate resistance training (RT) as the main therapy to positively modulate the morphological and functional parameters of older adults, once evidence indicate that this kind of exercise is able to maintain or even increase skeletal muscle mass, physical functionality, muscle strength, and power., ${ }^{5,6}$ submit your manuscript $\mid$ www.dovepress.com
Dovepress

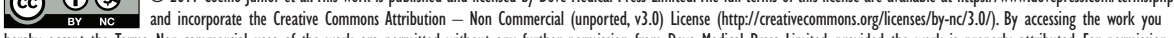
hereby accept the Terms. Non-commercial uses of the work are permitted without any further permission from Dove Medical Press Limited, provided the work is properly attributed. For permission for commercial use of this work, please see paragraphs 4.2 and 5 of our Terms (https://www.dovepress.com/terms.php). 
Moreover, the effects of RT go beyond those mentioned, as shown in a recent meta-analysis where positive changes in the hemodynamic parameters were observed after RT programs. ${ }^{7}$ Such benefits in the cardiovascular system are not exclusively derived from chronic adaptations once significant decreases in blood pressure values are commonly observed after a single bout of physical exercise - a phenomenon called post-exercise hypotension (PEH). ${ }^{8-14}$ These findings suggest that the chronic effects of RT can be a result of a sum of the acute effects. ${ }^{9}$ However, the optimum protocol of RT able to elicit PEH is still unknown, and most studies have been indicating beneficial results from traditional moderateintensity RT. ${ }^{10-12,14}$

Although the decrease in muscle strength is considered the most important neuromuscular alteration during aging, previous cross-sectional studies have demonstrated a higher decrease in muscle power capacity (the relation between strength and velocity of muscle contraction) than in muscle strength during aging. ${ }^{15}$ These data have high clinical relevance once muscle power is more associated with mobility and risk of falls than muscle strength. ${ }^{16}$

In this regard, recent guidelines from the ACSM advise that power training (PT) - characterized by a given movement or repetition performed as fast as possible at light to moderate loads - should be incorporated into physical exercise programs directed to older people. ${ }^{5}$ Regarding hemodynamic parameters, data have demonstrated that this kind of exercise has potential to cause PEH in young normotensive adults. ${ }^{17,18}$ However, to date, the acute effects of PT on hemodynamic parameters of older adults are still unknown.

Several mechanisms seem to be associated with $\mathrm{PEH}$, such as a better functioning of the cardiac and peripheral autonomic control and an increased bioavailability of vasoactive substances..$^{8,14,19}$ One of the most widely studied vasoactive substances is nitric oxide (NO). This molecule is a free radical with vasodilatory activity released by the vascular endothelium in response to several stimuli, including shear stress, which is generated by frictional resistance to blood flow on the vascular endothelial wall. ${ }^{19-21}$ During physical exercise, muscle contractions induce an increase in shear stress, consequently, increasing NO synthesis and releasing. ${ }^{19,21}$ Therefore, studies have been indicating NO as one possible mechanism associated with $\mathrm{PEH} .^{8,19,21}$ In addition, data have suggested that NO can be released after shear stress in a dose-dependent fashion. ${ }^{20}$ Thus, it is possible that concentric muscle contractions performed at high velocity during PT can cause greater NO bioavailability in comparison with the concentric muscle contractions performed at moderate velocity during RT, consequently, inducing larger PEH.

During aging, the bioavailability of NO is reduced, especially in women, and researchers indicate that the decreased estrogen synthesis found after menopause process has a key role in this phenomenon. ${ }^{22,23}$ Moreover, a recent meta-analysis indicated that women demonstrate a lower decrease in blood pressure values after acute physical exercise than men. ${ }^{24}$ Taken together, these data highlight the importance of studying the acute effects of exercise protocols on hemodynamic parameters and the bioavailability of NO in older women.

Therefore, in this study, we aimed to identify and compare the acute effects of a traditional moderate-intensity RT and a low-to-moderate intensity PT in the hemodynamic parameters and NO bioavailability of older women. Our hypothesis is that the high velocity of muscle contraction performed in PT could elicit greater shear stress and NO bioavailability causing higher PEH after PT than in RT, regardless of exercise intensity.

\section{Materials and methods}

We developed a randomized experimental study to elucidate and compare the acute effects of PT and RT on hemodynamic parameters and salivary NO bioavailability of normotensive and hypertensive older women. The methodology was based on the execution of 2 experimental sessions, an exercise session (PT or RT) and a control session (CS). The protocols of exercise were based on ACSM guidelines. ${ }^{5,6}$ Therefore, RT was based on 3 sets of $8-10$ repetitions of each exercise at the intensity of 5-6 on the adapted Borg scale. ${ }^{25}$ In turn, PT was also based on 3 sets of 8-10 repetitions of each exercise. However, PT group performed the session of exercise with a lower intensity -3 on the adapted Borg scale ${ }^{25}$ - than RT group. For quantification of $\mathrm{PEH}$, hemodynamic parameters were evaluated before and during 1 hour (immediately after [IA], and 5, 10, 15, 30, 45, and 60 minutes) after the exercise completion with the volunteers laid on an exercise mat. Furthermore, saliva was collected before and at 15, 30, and 60 minutes after exercise completion for NO quantification $(\mu \mathrm{M})$. Results obtained in this study will start to elucidate the acute effects of PT on hemodynamic parameters of older women, which are still unknown. Moreover, due to the performance of a second exercise group, it will be possible to compare the effects of this seminal protocol of PT with a traditional protocol of RT. Lastly, salivary NO quantification 
$(\mu \mathrm{M})$ will indicate a possible mechanism associated with $\mathrm{PEH}$ in older women.

\section{Subjects}

After approval of the study by the Research Ethics Committee of the University of Campinas (protocol no 835.733), and written informed consent being obtained from all participants, 21 untrained older women (age range 60-74 years) from 2 specialized public community health centers for older adults in a town of São Paulo city metropolitan area, in the southeastern Brazil, were recruited in 2015.

Diagnosis of hypertension (HTN) was not a criterion for exclusion. However, to ensure homogeneity, hypertensive $(n=10)$ and normotensive $(n=11)$ subjects were randomized separately. Therefore, hypertensive patients were first blindly allocated to PT or RT groups and, after, in the same way, normotensive subjects. In this sense, PT and RT groups were composed of $50 \%$ of hypertensive patients and $50 \%$ of normotensive subjects. For allocation of the participants in 1 of the 2 experimental groups (power or resistance), a computer-generated list of random numbers was used.

Subsequently, after performing the experimental sessions (PT and RT), 50\% of the volunteers of each group $(n=5)$ were randomized to the $\mathrm{CS}(\mathrm{n}=10)$ using a computer-generated list of random numbers. Anthropometric characteristics, hemodynamic variables, morbidity data, and the average number of medications used, as well as the class of medications, are shown in Table 1. Briefly, anthropometric characteristics (weight and height) were evaluated using a body weight scale with stadiometer Filizola ${ }^{\circledR}$ (BK50-FA, São Paulo, Brazil). In turn, use of medications and exclusion criteria data were collected from medical records (chart review) of each subject by 2 researchers (HJCJ and SSA). After data collection, the results were confronted to ensure reliability.

The diagnosis of HTN was accomplished by a specialist (cardiologist) who was not affiliated with the centers. In summary, before the participants began the activities in the centers where they were recruited, a medical consultation was conducted and an extensive list of medical examinations was required (fasting blood glucose, fasting blood insulin). If the patient showed any signal of HTN, such as elevated blood pressure levels along the day, she was referred to a specialist. After specific examinations, subjects should return to the centers with a letter signed by the specialist confirming or not the diagnosis. The final diagnosis was signed by the head physician of the center, and a nurse updated the medical records each 6 months.

Exclusion criteria were hormone replacement and/or psychotropic drugs use, cardiovascular disease (acute myocardial infarction, stroke, peripheral arterial disease, and transient ischemic disease), pulmonary disease, neurological or psychiatric disease (Parkinson's or Alzheimer's disease), musculoskeletal disorders, metabolic diseases (diabetes mellitus type II), comorbidities associated with greater risk of falls, and recent history of smoking or alcohol abuse. The participants had not been involved in RT or aerobic training in the last 12 months (untrained). ${ }^{26}$ Prior to any evaluation, a physician authorized volunteers to participate in the trial.

Table I Comparison between the groups in relation to morphological and cardiovascular variables

\begin{tabular}{|c|c|c|c|c|c|}
\hline Variables & Total $(n=2 I)$ & $\operatorname{CS}(n=10)$ & $\operatorname{RT}(n=\mid 0)$ & PT $(n=I I)$ & $P$-value \\
\hline Age (years) & $67.1 \pm 4.6$ & $66.8 \pm 5.4$ & $67.5 \pm 4.4$ & $66.7 \pm 4.7$ & 0.98 \\
\hline Height (m) & $1.58 \pm 0.0$ & $1.56 \pm 0.07$ & $1.56 \pm 0.05$ & $1.60 \pm 0.07$ & 0.55 \\
\hline BMI $\left(\mathrm{kg} / \mathrm{m}^{2}\right)$ & $28.0 \pm 4.9$ & $29.5 \pm 5.6$ & $30.3 \pm 4.0$ & $25.6 \pm 4.6$ & 0.27 \\
\hline SBP $(\mathrm{mmHg})$ & $135.1 \pm 2||$. & $129.8 \pm 11.9$ & $137.4 \pm 17.1$ & $133.1 \pm 23.4$ & 0.87 \\
\hline $\mathrm{DBP}(\mathrm{mmHg})$ & $83.6 \pm 16.0$ & $77.2 \pm 13.6$ & $87.5 \pm 21.5$ & $80.3 \pm 9.1$ & 0.55 \\
\hline MAP (mmHg) & $100.7 \pm 15.1$ & $94.7 \pm 11.8$ & $104.1 \pm 17.2$ & $97.9 \pm 12.8$ & 0.57 \\
\hline HR (bpm) & $77.5 \pm 13.0$ & $77.1 \pm I I .4$ & $79.3 \pm 11.8$ & $76.0 \pm 13.6$ & 0.95 \\
\hline DP (mmHg.bpm) & $10,474.3 \pm 2,335.5$ & $|0,038.0 \pm 1,9| 4.0$ & $10,880.8 \pm 1,990.0$ & $10,|4| .6 \pm 2,509.1$ & 0.85 \\
\hline HTN prevalence (\%) & $48 \%$ & $50 \%$ & $50 \%$ & $50 \%$ & ns \\
\hline Mean of medications & $0.62 \pm 0.80$ & $0.50 \pm 1.00$ & $0.70 \pm 0.78$ & $0.55 \pm 0.78$ & 0.96 \\
\hline \multicolumn{6}{|l|}{ Drug class (\%) } \\
\hline ANG II receptor antagonist & $80 \%$ & $80 \%$ & $80 \%$ & $80 \%$ & ns \\
\hline ACE inhibitor & $10 \%$ & $0 \%$ & $20 \%$ & $0 \%$ & ns \\
\hline Diuretic & $20 \%$ & $0 \%$ & $20 \%$ & $20 \%$ & ns \\
\hline Calcium channel blockers & $10 \%$ & $20 \%$ & $0 \%$ & $20 \%$ & ns \\
\hline
\end{tabular}

Note: Data presented as mean \pm standard deviation unless stated otherwise.

Abbreviations: CS, control session; RT, resistance training; PT, power training; BMI, body mass index; SBP, systolic blood pressure; DBP, diastolic blood pressure; MAP, mean arterial pressure; HR, heart rate; DP, double product; HTN, hypertension; ns, not significant; ANG, angiotensin; ACE, angiotensin-converting enzyme. 


\section{Procedures}

\section{Exercise protocol}

The exercise protocols were performed from July 2015 to December 2015. During all procedures, including physical training, the temperature in the laboratory was maintained between $21^{\circ} \mathrm{C}$ and $24^{\circ} \mathrm{C}$. Volunteers did not receive diet recommendations; however, they were required not to change their diet or food habits and the activities of daily living during the entire study period. The exercise protocols occurred under the supervision of 2 researchers (HJCJ and SSA), who were responsible for exercise prescription and monitoring of the exercise sessions.

Before the main sessions (power, strength, and control), volunteers were subjected to an adaptation period to avoid any bias related to the wrong execution of the exercises and to familiarize them with the Borg scale adapted by Foster et al, ${ }^{25}$ with the laboratory environment, and with the researchers. The adaptation period was the same for all subjects and involved the performance of the same exercise sessions in the morning (09:30 am-11:30 am), twice a week, with a minimum interval of 48 hours between each exercise bout, during 4 weeks. Each session was composed of 9 exercises, which were designed to stimulate the major muscle groups, at the intensity classified as easy in the adapted Borg scale, ${ }^{25}$ with 12-15 submaximal repetitions, avoiding fatigue, such that during the first week, volunteers performed 1 set of each exercise, and during the following weeks, they performed 2 sets of each exercise, with 1-minute rest interval between each set. ${ }^{5,6}$ Exercises were performed by alternating the major groups in the upper and lower extremities (alternating groups). The exercises that composed the adaptation period were 1) seated row, 2) leg press, 3) chest press, 4) seated leg curl, 5) lateral raise, 6) calf raise, 7) arm curl, 8) triceps extension, and 9) abdominal crunch. The duration of each exercise session was $\sim 50$ minutes. To avoid bias, elastic bands (Thera Band ${ }^{\circledR}$, Akron, OH, USA) were added in the exercise routine of the adaptation period. Therefore, exercises were performed exclusively not only with machines (Johnson Health Tech, Indaiatuba, SP, Brazil) and free weights but also with elastic bands (Thera Band).

During the adaptation period, the training load was adjusted by the rating of perceived exertion (RPE) method, using the adapted Borg scale. ${ }^{25,27}$ The volunteers received a plasticized copy of the adapted Borg scale ${ }^{25}$ after the end of each set and were asked the following question: "How much exertion did you make during the performance of this exercise set?" Volunteers could either mention the exertion score or indicate it on the scale. The RPE was utilized to increase the external validity of the data of this study since this method is reliable and easy to apply. Furthermore, this method allows the adjustment of the load in each session of exercise. Volunteers did not show differences in hemodynamic parameters before and after the end of the adaptation period (systolic blood pressure [SBP]: $P=0.35$; diastolic blood pressure [DBP]: $P=0.16$; mean arterial pressure [MAP]: $P=0.19$; heart rate [HR]: $P=0.97$; double product [DP]: $P=0.50$ ).

After the adaptation period, volunteers were blinded to treatment allocation and were randomized into the PT or RT group and, posteriorly, in CS, as aforementioned. Therefore, volunteers did not know in what treatment group they were allocated (power or resistance). Seventy-two hours before the start of the experimental protocols, hemodynamic variables were measured at rest (as described later in the "Hemodynamic measurements" section) and volunteers did not show significant differences in blood pressure values during rest (SBP: $P=0.87$; DBP: $P=0.55$; MAP: $P=0.57$; HR: $P=0.95$; DP: $P=0.85$; Table 1 ). Both the exercise groups (RT and PT) performed the same number of exercises and similar exercises aimed to stimulate the same muscle group. For this purpose, a same set of exercises was performed by the PT group using elastic bands (Thera Band) and by the RT group using machines (Johnson Health Tech) or free weights. The exercises performed were 1) squat on the chair $\left(90^{\circ}\right)$, 2) chest press, 3) seated leg curl, 4) frontal raise, 5) calf raise, 6) arm curl, 7) triceps extension, and 8) abdominal crunch. All exercises were performed in the total range of motion. In the beginning of every experimental session, all groups performed a brief warm-up that consisted of 1 set of 12-15 repetitions of each exercise without weights.

Both intervention groups were equalized by the total volume of the session (number of sets and repetitions); however, exercise intensity and muscle contraction velocity were established according to the peculiarities of each type of exercise, as recommended by the ACSM. ${ }^{5,6}$ An illustration of the methodological approach is shown in Figure 1.

\section{RT}

The exercise session of the RT group was based on 3 sets of 8-10 repetitions of each exercise, at the intensity of 5-6, which is considered difficult on the adapted Borg scale. ${ }^{25}$ This intensity represents $\sim 70 \%$ of 1 repetition maximum (1RM). ${ }^{27}$ One-minute rest interval was adopted between each set. Exercise cadence was 2-3 and 2 seconds for the concentric and eccentric phases, respectively. ${ }^{6,28}$ To ensure the cadence of muscle contractions, a researcher was responsible for counting the time and informing the volunteers during each 


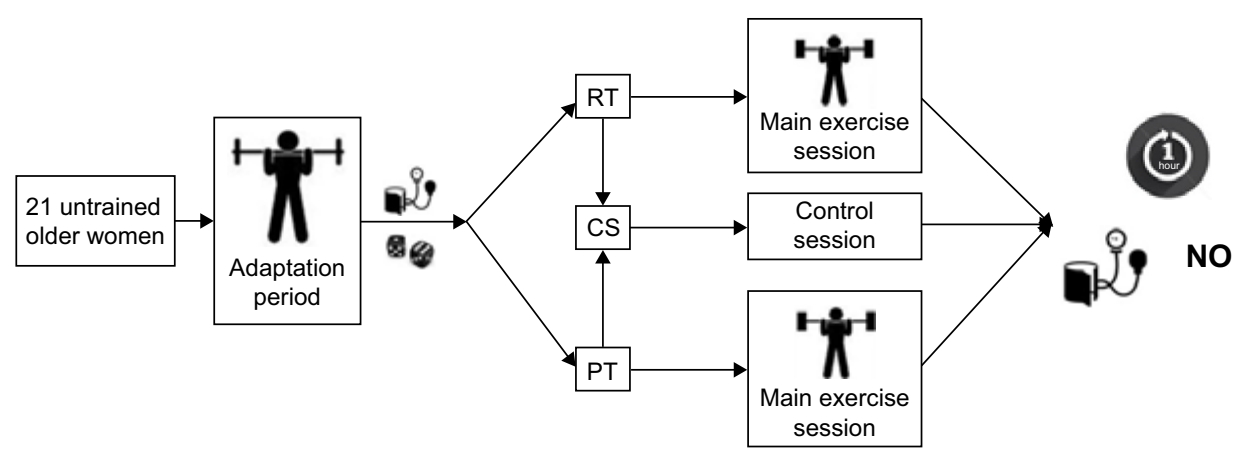

Figure I An illustration of the randomized experimental design used in this study.

Abbreviations: RT, resistance training; CS, control session; PT, power training; NO, nitric oxide.

muscle contraction, indicating if it was necessary to increase, decrease, or maintain the velocity.

\section{PT}

The exercise session of the PT group was based on 3 sets of 8-10 repetitions of each exercise, at the intensity of 3 , which is considered moderate on the adapted Borg scale. ${ }^{25}$ This intensity represents $\sim 50 \%$ of $1 \mathrm{RM} .{ }^{27}$ One-minute rest interval was adopted between each set. The concentric phase should be performed as fast as possible, whereas the eccentric phase should be performed within 2 seconds. ${ }^{6,28}$ To ensure the cadence of muscle contractions, a researcher was responsible for counting the time and informing the volunteers during each muscle contraction, indicating if it was necessary to increase, decrease, or maintain the velocity. In addition, before each concentric contraction, researchers stimulated the volunteers to perform the muscle contraction as fast as possible.

\section{CS}

During the CS, volunteers performed the same procedures of the experimental sessions. However, during the duration of an exercise session ( $\sim 50$ minutes), the study participants remained in a sitting position and could talk and read, but not drink, eat, or sleep. The CS was composed of $50 \%$ of the volunteers of each group, who were selected randomly. Therefore, a subset of the PT and RT groups composed the CS.

\section{Elastic bands}

The elastic bands were used to offer to the volunteers the possibility to perform the concentric contraction as fast as possible, avoiding any range-of-motion limitations imposed by the machines, since elastic bands propitiate large mobility in the achievement of the movement. ${ }^{29}$ Elastic bands are portable, inexpensive, reliable, and have high practical application. ${ }^{29,31}$ It is important to mention that the resistance of the elastic bands can be quantified in $\operatorname{kgf}(1 \mathrm{kgf}=1 \mathrm{~kg})$, thus allowing the quantification of the external load. ${ }^{29}$ Moreover, evidence has indicated similar morphological and functional adaptations after RT programs with equalized exercise intensity performed with machines or elastic bands. ${ }^{32}$ Therefore, the elastic bands were used as devices to create load, as machines and free weights, and should not be considered as an independent variable. Volunteers of this study performed the exercises using yellow and red elastic bands (Thera Band).

All the elastic bands were carefully used and, after, stored according to the manufacturer's directions (Thera Band).

\section{Total training workload and session rating of perceived exertion (SRPE)}

Total training workload was calculated using the formula: total number of sets $\times$ total number of repetitions $\times$ total weight lifted $(\mathrm{kg})$. In PT group, total weight lifted was calculated based on the elongation percentage of each elastic band, such as recommended by Uchida et al. ${ }^{30}$ To this, all elastic bands had, initially, the same height $(1 \mathrm{~m})$, and the extension in each exercise was measured individually during the adaptation period. The elongation performed by the volunteers was $\sim 100 \%$ of the initial height. To ensure the previously prescribed elongation, values were measured to total range of motion.

SRPE was quantified by SRPE method, ${ }^{27,33}$ wherein the volunteers rated the perceived exertion of the total exercise session. Ten minutes after the end of the exercise session, the study participants were asked the following question: "How much exertion did you make during the exercise session?"30, after which they chose a number in arbitrary units (AU), which was considered the SRPE. ${ }^{33}$ SRPE was quantified using the adapted Borg scale. ${ }^{25}$ 


\section{Hemodynamic measurements}

In the morning of the experiment, upon arriving at the laboratory (08:30 am-10:30 am), the participants were asked about any basic needs (drink water or use the bathroom) before the beginning of the experiments and were urged to avoid doing these activities during the experiments. Twenty minutes before the beginning and for 60 minutes after the end of the session of exercise, the volunteers remained lying on an exercise mat with their face up and eyes closed, without sleeping. The exercise room had an artificial light and automated control temperature $\left(24^{\circ} \mathrm{C}\right)$. Measurements of the hemodynamic parameters were performed blindly on the left arm using automated oscillometric equipment (BP 3BT0A; Microlife AG, Widnau, Switzerland) and were recorded in the 21 st minute after 20 minutes of rest (rest), IA ( 0 minute), and 5, 10, 15, 30, 45, and 60 minutes after the exercise completion. The hemodynamic parameters included SBP, DBP, and HR. The MAP and DP were determined using the following equations: $\mathrm{MAP}=$ systolic blood pressure $+(2 \times$ diastolic blood pressure $/ 3)$ and DP $=$ heart rate $\times$ systolic blood pressure. The procedures for the measurement of blood pressure were adapted from the VII Joint National Committee of High Blood Pressure (JNC7). ${ }^{34}$ All evaluations were performed by the same investigator.

\section{Saliva collection}

Saliva samples were collected at rest, immediately before the exercises, and at 15, 30, and 60 minutes after the end of the exercise using a roller cotton (Cremer, São Paulo, Brazil). Researchers asked the participants to put a piece of cotton in their mouth and remove it when it became soggy. The samples were transferred to a Falcon tube and frozen immediately until the end of the experiment. In the laboratory, the samples were centrifuged and supernatants were stored at $-80^{\circ} \mathrm{C}$ for posterior analyses using the Griess colorimetric method. ${ }^{21}$

\section{Measurement of $\mathrm{NO}$}

The NO pool was considered the mensuration of nitrite $\left(\mathrm{NO}_{2}^{-}\right)$levels in saliva. Briefly, a solution containing $0.1 \%$ $\mathrm{N}$-(1-naphthyl)-ethylenediamine (NED) (Sigma-Aldrich Co., St Louis, MO, USA) and 1.0\% sulfanilamide (Sigma) was prepared in $2.5 \%$ phosphoric acid as the diluent. Saliva samples $(50 \mu \mathrm{L})$ and Griess reagent $(50 \mu \mathrm{L})$ were mixed and transferred to microplates. Absorbance was measured at $530 \mathrm{~nm}$, and sodium nitrite $\left(\mathrm{NaNO}_{2}\right)$ was used as the standard. Nitrite quantification ( $\mu \mathrm{M})$ was achieved using a standard curve constructed with $\mathrm{NaNO}_{2}$ at the concentrations of 100 ,
$50,25,12.5,6.25,3.13,1.66$, and $0 \mu \mathrm{M} .{ }^{35}$ Data were analyzed using the Microplate Software (Hercules, CA, USA).

\section{Statistical analyses}

Normality of data was tested using the Kormonov-Smirnov test. Mann-Whitney test was applied to verify differences in the SRPE and total training workload between the groups. Differences in the percentage of hypertensive volunteers between the groups were calculated using $\chi^{2}$ test. Comparisons between the groups for age, height, body mass index (BMI), SBP, DBP, MAP, HR, DP, and average number of medications was performed using one-way analysis of variance (ANOVA) followed by Dunnett's post-hoc test. Intragroup and intergroup comparisons in the different periods (rest, $0,5,10,15,30,45$, and 60 minutes after the end of each session) for SBP, DBP, MAP, HR, DP, and NO were performed using split-plot ANOVA followed by Dunnett's posthoc test. Cohen's effect size (ES) $d$ was calculated to assess the magnitude of the results. The ES was classified according to Rhea. ${ }^{26}$ The level of significance was $5 \%(P<0.05)$, and all procedures were performed using the Statistical Package for the Social Sciences software (IBM Corp, Armonk, NY, USA). The power of the sample size was determined using $G^{*}$ Power version 3.1.9.2 on the basis of the magnitude of the mean differences in the 6 variables between the 3 groups. The ES required for a sample size of 15 volunteers with a level of significance set at $5 \%$ and power $(\beta)$ of 0.80 was 1.0 (larger ES). All values are shown as mean $\pm \mathrm{SD}$, except for SRPE which is shown as median $\pm \mathrm{SD}$.

\section{Results}

Table 1 shows the morphological and hemodynamic characteristics of older women recruited in this study. No significant differences in these characteristics were observed between the groups. Regarding pharmacological treatment, the mean of medications used by the volunteers and the percentage of drug class were not different between the groups. Volunteers' attendance was $100 \%$ for all experimental sessions (exercise and control). Furthermore, volunteers did not present adverse effects during or after the experimental sessions.

During the training sessions, as expected, the PT group showed a lower perception of effort, indicated through the

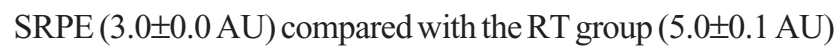
$(P<0.001)$. Moreover, even if the total number of sets and repetitions were equalized between the groups, total training workload (total number of sets $\times$ total number of repetitions $\times$ total weight lifted [kg]) was higher in RT group $(86,050 \pm 4,550 \mathrm{~kg})$ than that in PT group $(16,330 \pm 208 \mathrm{~kg})(P<0.001)$. 
Post-exercise data for SBP, DBP, MAP, HR, and DP are shown in Table 2. SBP in the PT group decreased at 10, 15,30 , and 45 minutes after the end of the exercise session ( $F=3.7 ; P=0.001)$ compared with the rest period in the same group (Table 2). The ES in the PT group was classified as small at 15 (Cohen's $d=0.81$ ), 30 (Cohen's $d=0.91$ ), and 45 minutes (Cohen's $d=1.07$ ) and moderate at 60 minutes

Table 2 Behavior of hemodynamic parameters after the experimental sessions

\begin{tabular}{|c|c|c|c|}
\hline Variables & CS & RT & PT \\
\hline \multicolumn{4}{|l|}{ SBP } \\
\hline IA & $-4.3 \pm 7.0$ & $-0.3 \pm 12.0$ & $0.4 \pm 8.1$ \\
\hline $5^{\prime \prime}$ & $2.1 \pm 14.9$ & $-3.1 \pm 11.9$ & $-6.1 \pm 6.7$ \\
\hline $10^{\prime \prime}$ & $3.6 \pm 19.5$ & $-6.4 \pm 11.9$ & $-7.8 \pm 8.0 *$ \\
\hline $15^{\prime \prime}$ & $8.1 \pm 26.9$ & $-1.0 \pm 4.9$ & $-7.3 \pm 7.4^{*}$ \\
\hline $30^{\prime \prime}$ & $4.1 \pm 19.4$ & $-2.4 \pm 4.6$ & $-9.1 \pm 10.0 * *$ \\
\hline $45^{\prime \prime}$ & $4.0 \pm 13.8$ & $-4.8 \pm 11.2$ & $-7.5 \pm 10.2^{*}$ \\
\hline $60^{\prime \prime}$ & $2.6 \pm 16.5$ & $-3.9 \pm 8.8$ & $-5.1 \pm 8.4$ \\
\hline \multicolumn{4}{|l|}{ DBP } \\
\hline IA & $-7.3 \pm 6.9$ & $0.8 \pm 8.1$ & $3.0 \pm 6.4$ \\
\hline $5^{\prime \prime}$ & $-1.9 \pm 18.5$ & $-0.3 \pm 9.3$ & $1.1 \pm 8.0$ \\
\hline $10^{\prime \prime}$ & $5.1 \pm 14.5$ & $-0.7 \pm 9.4$ & $-3.2 \pm 9.9$ \\
\hline $15^{\prime \prime}$ & $1.5 \pm 14.9$ & $-2.3 \pm 7.8$ & $1.6 \pm 8.1$ \\
\hline $30^{\prime \prime}$ & $0.0 \pm 14.7$ & $-8.4 \pm 30.7$ & $1.7 \pm 10.1$ \\
\hline $45^{\prime \prime}$ & $2.3 \pm 15.9$ & $-1.7 \pm 9.9$ & $-0.5 \pm 8.0$ \\
\hline $60^{\prime \prime}$ & $4.8 \pm 10.8$ & $-1.4 \pm 10.1$ & $-0.2 \pm 7.9$ \\
\hline \multicolumn{4}{|l|}{ MAP } \\
\hline IA & $-6.3 \pm 6.2$ & $0.8 \pm 5.7$ & $2.4 \pm 7.3$ \\
\hline $5^{\prime \prime}$ & $5.8 \pm 14.0$ & $-1.0 \pm 8.2$ & $-1.1 \pm 6.7$ \\
\hline $10^{\prime \prime}$ & $5.1 \pm 13.2$ & $-2.6 \pm 8.0$ & $-4.6 \pm 9.1$ \\
\hline $15^{\prime \prime}$ & $-1.0 \pm 11.0$ & $-2.8 \pm 7.3$ & $-1.3 \pm 7.1$ \\
\hline $30^{\prime \prime}$ & $-2.4 \pm 10.6$ & $-6.8 \pm 20.8$ & $-1.8 \pm 9.7$ \\
\hline $45^{\prime \prime}$ & $1.4 \pm 3.0$ & $-2.9 \pm 7.6$ & $-2.8 \pm 8.7$ \\
\hline $60^{\prime \prime}$ & $1.1 \pm 6.3$ & $-2.1 \pm 8.3$ & $-1.3 \pm 9.2$ \\
\hline \multicolumn{4}{|l|}{$\mathrm{HR}$} \\
\hline IA & $-3.8 \pm 20.4$ & $-14.2 \pm 29.7^{*}$ & $3.1 \pm 7.4$ \\
\hline $5^{\prime \prime}$ & $1.3 \pm 21.8$ & $-14.8 \pm 27.7^{*}$ & $1.9 \pm 8.3$ \\
\hline $10^{\prime \prime}$ & $1.5 \pm 10.3$ & $-15.3 \pm 26.9 * *$ & $-1.3 \pm 4.1$ \\
\hline $15^{\prime \prime}$ & $-2.8 \pm 12.3$ & $-15.4 \pm 27.5 * *$ & $-2.2 \pm 4.6$ \\
\hline $30^{\prime \prime}$ & $-1.8 \pm 10.2$ & $-16.2 \pm 27.3 * *$ & $-4.6 \pm 4.8$ \\
\hline $45^{\prime \prime}$ & $-0.3 \pm 6.4$ & $-18.8 \pm 26.4^{* * *}$ & $-4.7 \pm 3.2$ \\
\hline $60^{\prime \prime}$ & $2.5 \pm 8.0$ & $-19.2 \pm 26.1 * * *$ & $-9.9 \pm 18.0^{*}$ \\
\hline \multicolumn{4}{|l|}{ DP } \\
\hline IA & $-1,792 \pm 3,318$ & $48 I \pm I, 062$ & $-769 \pm 2,609$ \\
\hline $5^{\prime \prime}$ & $-2,110 \pm 3,423$ & $-390 \pm 638$ & $-338 \pm 1,186$ \\
\hline $10^{\prime \prime}$ & $-2,482 \pm 3,327$ & $-748 \pm 663^{* * *}$ & $8 I \pm 2,63 \mid$ \\
\hline $15^{\prime \prime}$ & $-2,338 \pm 3,286$ & $-802 \pm 552$ & $37 \pm 3,120$ \\
\hline $30^{\prime \prime}$ & $-2,336 \pm 3,297$ & $-1,264 \pm I, 044$ & $-449 \pm 1,540$ \\
\hline $45^{\prime \prime}$ & $-2,797 \pm 3,240$ & $-1,065 \pm 517^{* * *}$ & $-5 \mid 4 \pm 1,608$ \\
\hline $60^{\prime \prime}$ & $-2,7 \mid 4 \pm 3,125$ & $-I, 54 I \pm 2,667 * * * *$ & $-28 I \pm I, 447 *$ \\
\hline
\end{tabular}

Notes: $* P<0.05$ vs baseline data prior to exercise; $* * P<0.01$ vs baseline data prior to exercise; ${ }^{* * * P}<0.001$ vs baseline data prior to exercise. Data presented as mean \pm standard deviation.

Abbreviations: CS, control session; RT, resistance training; PT, power training; SBP, systolic blood pressure; IA, immediately after; DBP, diastolic blood pressure; MAP, mean arterial pressure; HR, heart rate; DP, double product.
(Cohen's $d=1.40$ ) after exercise completion (Table 3). On the other hand, intragroup comparisons did not demonstrate significant differences in SBP on RT group and CS. In addition, DBP and MAP (Table 2) did not change significantly in the RT, PT, and CS.

The RT group showed a significant decrease in HR postexercise during the entire evaluation period compared with the rest period $(F=3.4 ; P<0.001)$ (Table 2$)$. By contrast, a

Table 3 ES and its classification of behavior of hemodynamic parameters after the experimental sessions

\begin{tabular}{|c|c|c|c|}
\hline Variables & CS & RT & PT \\
\hline \multicolumn{4}{|l|}{ SBP } \\
\hline IA & 0.40 (trivial) & -0.43 (trivial) & -0.54 (small) \\
\hline $5^{\prime \prime}$ & -0.15 (trivial) & 0.30 (trivial) & 0.72 (small) \\
\hline $10^{\prime \prime}$ & -0.26 (trivial) & 0.67 (small) & 0.78 (small) \\
\hline $15^{\prime \prime}$ & -0.40 (trivial) & 0.55 (small) & $0.8 \mathrm{I}$ (small) \\
\hline $30^{\prime \prime}$ & -0.26 (trivial) & 0.48 (trivial) & 0.91 (small) \\
\hline $45^{\prime \prime}$ & -0.32 (trivial) & 0.74 (small) & I.07 (small) \\
\hline $60^{\prime \prime}$ & -0.22 (trivial) & 0.49 (trivial) & I. 40 (moderate) \\
\hline \multicolumn{4}{|l|}{ DBP } \\
\hline IA & 0.66 (small) & -1.19 (small) & -1.6 (moderate) \\
\hline $5^{\prime \prime}$ & 0.12 (trivial) & -0.15 (trivial) & -0.2 (trivial) \\
\hline $10^{\prime \prime}$ & -0.36 (trivial) & 0.43 (trivial) & 0.67 (small) \\
\hline $15^{\prime \prime}$ & -0.14 (trivial) & 0.36 (trivial) & 0 (trivial) \\
\hline $30^{\prime \prime}$ & 0 (trivial) & 0.72 (small) & -0.16 (trivial) \\
\hline $45^{\prime \prime}$ & -0.13 (trivial) & 0.24 (trivial) & 0.25 (trivial) \\
\hline $60^{\prime \prime}$ & $-0.4 \mathrm{I}$ (trivial) & 0.63 (small) & 0.64 (small) \\
\hline \multicolumn{4}{|l|}{ MAP } \\
\hline IA & 0.64 (small) & -1.08 (small) & -1.33 (moderate \\
\hline $5^{\prime \prime}$ & -0.59 (small) & -0.47 (trivial) & -0.49 (trivial) \\
\hline $10^{\prime \prime}$ & -0.39 (trivial) & $0.7 \mathrm{I}$ (small) & 0.98 (small) \\
\hline $15^{\prime \prime}$ & 0 (trivial) & 0.12 (trivial) & 0 (trivial) \\
\hline $30^{\prime \prime}$ & 0.17 (trivial) & 0.26 (trivial) & 0 (trivial) \\
\hline $45^{\prime \prime}$ & -0.10 (trivial) & 0.55 (small) & 0.74 (small) \\
\hline $60^{\prime \prime}$ & 0 (trivial) & 0.46 (trivial) & 0.46 (trivial) \\
\hline \multicolumn{4}{|l|}{$\mathrm{HR}$} \\
\hline IA & 0 (trivial) & 0.43 (trivial) & -0.48 (trivial) \\
\hline $5^{\prime \prime}$ & 0 (trivial) & 0.64 (small) & 0 (trivial) \\
\hline $10^{\prime \prime}$ & -0.18 (trivial) & 0.89 (small) & 2.23 (large) \\
\hline $15^{\prime \prime}$ & 0.25 (trivial) & 0.59 (small) & -0.1 I (trivial) \\
\hline $30^{\prime \prime}$ & 0.18 (trivial) & 0.73 (small) & 0.39 (trivial) \\
\hline $45^{\prime \prime}$ & 0 (trivial) & I.02 (small) & I.05 (small) \\
\hline $60^{\prime \prime}$ & -0.31 (trivial) & I.24 (small) & I.05 (small) \\
\hline \multicolumn{4}{|l|}{ DP } \\
\hline IA & 0.35 (trivial) & 0.36 (trivial) & -0.68 (small) \\
\hline $5^{\prime \prime}$ & 0.21 (trivial) & 0.73 (small) & -0.14 (trivial) \\
\hline $10^{\prime \prime}$ & 0 (trivial) & 0.90 (small) & 0.40 (trivial) \\
\hline $15^{\prime \prime}$ & 0 (trivial) & 0.78 (small) & 0.40 (trivial) \\
\hline $30^{\prime \prime}$ & 0.22 (trivial) & 0.77 (small) & 0.66 (small) \\
\hline $45^{\prime \prime}$ & 0.25 (trivial) & 0.94 (small) & 0.55 (small) \\
\hline $60^{\prime \prime}$ & 0.14 (trivial) & I.06 (small) & 0.48 (trivial) \\
\hline
\end{tabular}

Note: Data presented as Cohen's $d$

Abbreviations: ES, effect size; CS, control session; RT, resistance training; PT, power training; SBP, systolic blood pressure; IA, immediately after; DBP, diastolic blood pressure; MAP, mean arterial pressure; HR, heart rate; DP, double product. 
significant decrease in HR was observed only 60 minutes after the end of the exercise session in the PT group (Table 2). A trivial ES was observed IA the end of the exercise session, and a small ES was observed in the other measurements (5, $10,15,30,45$, and 60 minutes after the end of the session) in the RT group (Table 3). In turn, PT group presents a larger number of trivial classifications (4/7): IA and at 5, 15, and 30 minutes of the end of the session (Table 3 ).

DP decreased at 10, 45, and 60 minutes after the exercise sessions in the RT group compared with the rest period ( $F=4.7 ; P<0.001$ ) (Cohen's $d=0.90,0.94$, and 1.06, respectively) but decreased only at 60 minutes after the end of the session in the PT group (Cohen's $d=0.69$ ) (Tables 2 and 3).

Results from the statistical analyses of the intergroup comparisons $(\mathrm{PT} \times \mathrm{RT} \times \mathrm{CS})$ did not show significant differences regarding SBP, DBP, MAP, HR, and DP.

Table 4 shows the cardiovascular parameters in the rest period and the mean of 1-hour post-exercise. The RT group showed decreased HR (Cohen's $d=2.1$ ) and DP (Cohen's $d=2.2$ ) compared with the PT group and CS. The PT group had a significant decrease in SBP 1 hour

Table 4 Comparison of the rest and the mean of the moments after the exercise

\begin{tabular}{|c|c|c|c|}
\hline Variables & CS & RT & PT \\
\hline \multicolumn{4}{|l|}{$\overline{S B P}$} \\
\hline Rest & $129.8 \pm 4.2$ & $127.4 \pm 5.2$ & $133.5 \pm 5.5$ \\
\hline$\Delta \mathrm{I}$ hour & $2.9 \pm 3.8$ & $-3.4 \pm 3.5$ & $-6.9 \pm 2.6^{* \neq \neq}$ \\
\hline Intragroup $P$-value & 0.54 & 0.35 & 0.02 \\
\hline ES (classification) & -0.72 (small) & 0.7 (small) & I.5 (moderate) \\
\hline \multicolumn{4}{|l|}{ DBP } \\
\hline Rest & $77.2 \pm 4.8$ & $77.7 \pm 2.9$ & $76.9 \pm 3.8$ \\
\hline$\Delta \mathrm{I}$ hour & $0.6 \pm 4.1$ & $-2.0 \pm 3.8$ & $-0.3 \pm 2.5$ \\
\hline Intragroup $P$-value & 0.87 & 0.61 & 0.89 \\
\hline ES (classification) & -0.13 (trivial) & 0.4 (trivial) & 0 (trivial) \\
\hline \multicolumn{4}{|l|}{ MAP } \\
\hline Rest & $94.7 \pm 4.1$ & $94.3 \pm 3.0$ & $95.7 \pm 4.1$ \\
\hline$\Delta \mathrm{I}$ hour & $0.5 \pm 0.4$ & $-2.4 \pm 2.7$ & $-2.5 \pm 2.4$ \\
\hline Intragroup $P$-value & 0.83 & 0.39 & 0.32 \\
\hline ES (classification) & -0.17 (trivial) & 0.5 (small) & 0.5 (small) \\
\hline \multicolumn{4}{|l|}{ HR } \\
\hline Rest & $77.1 \pm 4.0$ & $85.8 \pm 6.4$ & $73.3 \pm 2.3$ \\
\hline$\Delta \mathrm{I}$ hour & $-0.4 \pm 0.4$ & $-16.2 \pm 9.0^{\# . \neq}$ & $-2.5 \pm 1.2$ \\
\hline Intragroup $P$-value & 0.28 & 0.10 & 0.07 \\
\hline ES (classification) & -0.14 (trivial) & 2.I (large) & 0.5 (small) \\
\hline \multicolumn{4}{|l|}{ DP } \\
\hline Rest & $10,038.1 \pm 676.9$ & $11,000.1 \pm 1,026.7$ & $9,786.0 \pm 5 \mid 7.2$ \\
\hline$\Delta \mathrm{I}$ hour & $-318.9 \pm 557.5$ & $-2,367.0 \pm 1,086.0^{\ddagger} . \$$ & $-693.6 \pm 181.9 *$ \\
\hline Intragroup $P$-value & 0.51 & 0.06 & 0.00 \\
\hline ES (classification) & 0.5 (small) & 2.2 (large) & I.7 (moderate) \\
\hline
\end{tabular}

Notes: $* P<0.05$ vs rest; $\$ P<0.05$ vs control; $\$ P<0.05 ; ~ * P<0.05$ vs $P$ T. Data presented as mean \pm standard deviation and Cohen's $d(E S)$.

Abbreviations: CS, control session; RT, resistance training; PT, power training; SBP, systolic blood pressure; ES, effect size; DBP, diastolic blood pressure; MAP, mean arterial pressure; HR, heart rate; DP, double product. after the training session (Cohen's $d=1.5$ ) compared with the rest period and the CS and a significant decrease in DP (Cohen's $d=1.7$ ) compared with the rest period. However, these changes on SBP were not observed in the RT group.

To evaluate the possible relationship of PEH in the PT group, NO was measured to assess its potential involvement. The RT and PT groups demonstrated a significant increase in NO levels 60 minutes after the end of the session compared with the rest period. Despite the apparent greater bioavailability of NO in the PT, NO levels were similar among the groups (Figure 2).

\section{Discussion}

The main finding of this study is that an acute session of PT can cause a significant decrease in SBP and DP in older women. In turn, data did not indicate significant PEH after RT; however, HR and DP were decreased in the RT group after exercise completion. Moreover, both kinds of exercise were equally effective to increase salivary NO bioavailability.

Regarding PT results, data indicate a significant decrease in SBP ( - $7.9 \mathrm{mmHg})$ during 35 minutes after exercise completion (from the 10 th minute to the 45 th minute), consequently, characterizing PEH. These data are similar to other studies, which observed $\mathrm{PEH}$ after resistance and endurance exercises..$^{8,10-14,19,21}$ To the best of our knowledge, this is the first study designed to investigate the acute effects of PT on hemodynamic parameters of older women, thereby limiting data discussion. However, the amount of evidence demonstrating beneficial hemodynamic effects after explosive-type strength training in young normotensive adults have been increasing exponentially. ${ }^{17,18}$

In fact, recently, Arazi et $\mathrm{al}^{17}$ and Ramírez-Campillo et al ${ }^{18}$ investigated healthy young physically active normotensive

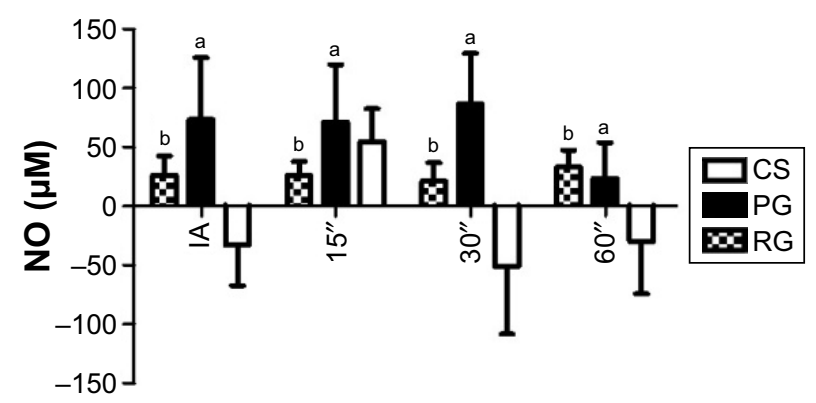

Figure 2 Mean and standard deviation values of $\mathrm{NO}$ after the end of the exercise session.

Notes: ${ }^{\mathrm{a}} \mathrm{P}<0.05=\mathrm{PG}$ vs baseline data prior to exercise; ${ }^{\mathrm{b}} \mathrm{P}<0.05=\mathrm{RG}$ vs baseline data prior to exercise.

Abbreviations: NO, nitric oxide; PG, power group; RG, resistance group; CS, control session. 
subjects in a crossover design protocol composed of 3 different sessions of acute plyometric training: low intensity, moderate intensity, and high intensity. Results of both studies demonstrated that all protocols of plyometric training, regardless of exercise intensity, were equally effective to elicit PEH. ${ }^{17,18}$ Moreover, the magnitude of the decrease in SBP showed after the session of exercise with low $(\sim-7 \mathrm{mmHg})$ and moderate ( -9 mmHg) intensities was similar with this study. ${ }^{18}$

Therefore, taken together, data indicate that explosivetype strength training is not exclusively effective to cause $\mathrm{PEH}$ in healthy young normotensive adults but also can promote significant decrease in the SBP of hypertensive and normotensive older women, indicating that the muscle's contraction velocity can be a factor involved in $\mathrm{PEH}$, such as other variables of exercise training, such as intensity, volume, and order of exercises.

On the other hand, in this investigation, RT did not show significant PEH, whereas there was a significant decrease in HR and DP during the whole period of recovery and in the mean of 1 hour after exercise (Table 4). Indeed, the optimal resistance exercise protocol to elicit $\mathrm{PEH}$ is yet unknown, and the results of previous studies are controversial. ${ }^{11-14}$ However, in contrast to our results, moderate-intensity RT has been shown to be effective in causing $\mathrm{PEH}$ without significant changes in HR and/or DP. ${ }^{11-14}$

Mota et al, ${ }^{13}$ for example, evaluated a sample similar to ours and did not observe PEH after a single low-intensity resistance exercise session. However, when volunteers were subjected to a higher intensity ( $70 \%$ of $1 \mathrm{RM})$ resistance exercise session, $\mathrm{PEH}$ was observed during the whole evaluation period after the end of the exercise session. ${ }^{13}$ Recently, Gerage et $\mathrm{al}^{12}$ evaluated the blood pressure levels after an acute session of sub-maximal resistance exercise in older women and found a moderate decrease $(-5 \mathrm{mmHg})$ in SBP 1 hour after the end of the exercise. Furthermore, data in the literature have demonstrated decreased cardiac autonomic modulation after RT protocols, which inhibits the decrease in HR after exercise. ${ }^{10,14,36}$ Therefore, dissimilar results between protocols can be explained by differences in the variables of RT (volume and number of exercises).

Several mechanisms have been indicated to be responsible for PEH, such as an ameliorated cardiac and peripheral autonomic control, as well as an increased bioavailability of vasoactive substances (eg, NO). ${ }^{8,14,19}$ Salivary NO was investigated in this study as a possible modulator of $\mathrm{PEH}$, and data demonstrate increased salivary NO bioavailability the whole time after exercise completion (60 minutes) in comparison with rest moment in PT and RT.
Regarding PT, data of this study are in concordance with other experiments, which have observed that plasma and salivary NO were associated with $\mathrm{PEH}$ after resistance and aerobic exercises. ${ }^{8,19,21}$ Interestingly, different from PT, increased NO bioavailability in RT was not able to induce $\mathrm{PEH}$. On the contrary, the lack of PEH in RT is possibly associated with increased peripheral vascular resistance, since cardiac output is probably decreased due to reduced HR. Moreover, even if evidence has been demonstrating that NO can change autonomic modulation in rats and humans, ${ }^{37-39}$ inducing ameliorated parasympathetic control of the heart, which could explain data observed in RT, more studies are necessary to elucidate the mechanisms responsible for the effects of PT and RT in the cardiovascular system.

Some limitations of this study should be mentioned to collaborate with better inferences about the data. Regarding the exercise prescription, PT and RT performed exercise protocols with different intensities, which limit comparison between both protocols. Therefore, from this seminal data, future studies have been developed in order to compare the effects of equalized-intensity programs of resistance and power exercise on hemodynamic parameters.

Furthermore, the study sample was composed of a mix of hypertensive and normotensive subjects, including the CS. It is well accepted that hypertensive subjects present higher $\mathrm{PEH}$ than normotensive subjects, ${ }^{12,40}$ thereby it is possible that some hypertensive subjects of PT showed higher PEH in comparison with RT. In an attempt to reduce such limitation and increase inferences, we performed a new series of analyses only with hypertensive patients ( $n=5$ in each group) (Tables S1-S3). Data indicate that, in hypertensive patients, data were similar to those observed in the larger sample composed by hypertensive and normotensive subjects. Therefore, data of this study can be a product only of the hypertensive patients. However, sample size avoids larger inferences.

In addition, even if there were no differences regarding BMI values among the groups, a slightly higher BMI was observed in the RT group. Once BMI can be associated with several mechanisms indicated to be responsible for high blood pressure values, as well as disruptions in the main pathways responsible for $\mathrm{PEH}$, future studies should consider samples composed exclusively by hypertensive and normotensive subjects with normal or high BMI to confirm the data of this study.

Lastly, our data contribute with strength and conditioning coaches, as well as with physiotherapists, gerontologists, sports physicians, and occupational therapists who work with physical exercise and older people once data demonstrated 
that beneficial effects of physical exercise in the hemodynamic parameter of older people are not only limited to aerobic training and RT but also can be observed after PT programs with elastic band, which increases the external validity of data of this study since healthy professionals can serve customers and patients in their homes and a specific place is not necessary.

\section{Conclusion}

The seminal data of this study indicate that an acute session of PT and resistance exercise can be effective to cause beneficial changes on hemodynamic parameters and NO levels of older women. Moreover, ES classification suggests that PT can be superior than RT to elicit decrease on SBP, indicating the potential of this kind of exercise training to be used as non-pharmacological tool in the treatment of HTN.

\section{Acknowledgments}

The authors are grateful to the Coordenação de Aperfeiçoamento de Pessoal de Nível Superior (CAPES) and Conselho Nacional de Desenvolvimento Científico e Tecnológico (CNPq) for funding this research via scholarships to HJCJ and RYA, respectively. BR and MCCI had financial support from the Fundação de Amparo à Pesquisa do Estado de São Paulo (FAPESP) and CNPq (BPQ). The authors are also grateful to Daisy dos Reis and Flávio Romano of the facility for older adults and all researchers of the Research Group on Chronic-Degenerative Diseases of Mogi das Cruzes University (Grupo de Pesquisa em Doenças Crônico-Degenerativas da Universidade de Mogi das Cruzes-GEDCD/UMC) for their support. The authors alone are responsible for the content and writing of the paper.

\section{Disclosure}

The authors report no conflicts of interest in this work.

\section{References}

1. Morley JE, Abbatecola AM, Argiles JM, et al. Sarcopenia with limited mobility: an international consensus. J Am Med Dir Assoc. 2011; 12(6):403-409.

2. Cruz-Jentoft AJ, Baeyens JP, Bauer JM, et al. Sarcopenia: European consensus on definition and diagnosis: report of the European Working Group on sarcopenia in older people. Age Ageing. 2010;39:412-423.

3. Coelho Júnior HJ, Aguiar Sda S, Gonçalves Ide O, et al. Sarcopenia is associated with high pulse pressure in older women. J Aging Res. 2015;2015:109824.

4. Kim KS, Park KS, Kim MJ, Kim SK, Cho YW, Park SW. Type 2 diabetes is associated with low muscle mass in older adults. Geriatr Gerontol Int. 2014;14:115-121.

5. Chodzko-Zajko WJ, Proctor DN, Fiatarone Singh MA, et al. American College of Sports Medicine position stand. Exercise and physical activity for older adults. Med Sci Sports Exerc. 2009;41: $1510-1530$.
6. Garber CE, Blissmer B, Deschenes MR, et al; American College of Sports Medicine. American College of Sports Medicine position stand. Quantity and quality of exercise for developing and maintaining cardiorespiratory, musculoskeletal, and neuromotor fitness in apparently healthy adults: guidance for prescribing exercise. Med Sci Sports Exerc. 2011;43:1334-1359.

7. Cornelissen VA, Smart NA. Exercise training for blood pressure: a systematic review and meta-analysis. J Am Heart Assoc. 2013;2: e004473.

8. Asano RY, Browne RAV, Sotero RDC, et al. Cycling above is more effective than below lactate threshold for nitric oxide release and postexercise blood pressure reduction in individuals with type-2 diabetes. Motriz. 2013;19(3):633-640.

9. Terra DF, Mota MR, Rabelo HT, et al. Redução da pressão arterial e do duplo produto de repouso após treinamento resistido em idosas hipertensas [Reduction of arterial pressure and double product at rest after resistance exercise training in elderly hypertensive women]. Arq Bras Cardiol. 2008;91(5):299-305.

10. Lima AH, Forjaz CL, Silva GQ, Menêses AL, Silva AJ, Ritti-Dias RM. Acute effect of resistance exercise intensity in cardiac autonomic modulation after exercise. Arq Bras Cardiol. 2011;96:498-503.

11. Macedo M, Silva A, Olher R, et al. Post-exercise hypotension between different protocols of resistance training for beginners. $J$ Exercise Physiol. 2014:17(6):58-66.

12. Gerage AM, Ritti-Dias RM, do Nascimento MA, et al. Chronic resistance training does not affect post-exercise blood pressure in normotensive older women: a randomized controlled trial. Age. 2015;37:1-10.

13. Mota MR, de Oliveira RJ, Dutra MT, et al. Acute and chronic effects of resistive exercise on blood pressure in hypertensive elderly women. J Strength Cond Res. 2013;27:3475-3480.

14. Figueiredo T, Willardson J, Miranda H, et al. Influence of load intensity on post-exercise hypotension and heart rate variability following a strength training session: exercise intensity and cardiovascular response. J Strength Cond Res. 2015;29(10):2941-2948.

15. Dietzel R, Gast U, Heine T, Felsenberg D, Armbrecht G. Cross-sectional assessment of neuromuscular function using mechanography in women and men aged 20-85 years. J Musculoskelet Neuronal Interact. 2013;13:312-319.

16. Bean JF, Leveille SG, Kiely DK, Bandinelli S, Guralnik JM, Ferrucci L. A comparison of leg power and leg strength within the InCHIANTI study: which influences mobility more? J Gerontol A Biol Sci Med Sci. 2003;58:M728-M733.

17. Arazi H, Asadi A, Rahimzadeh M, Moradkhani AH. Post-plyometric exercise hypotension and heart rate in normotensive individuals: influence of exercise intensity. Asian J Sports Med. 2013;4(4):235-240.

18. Ramírez-Campillo R, Abad-Colil F, Vera M, et al. Men and women exhibit similar acute hypotensive responses after low, moderate, or high-intensity plyometric training. J Strength Cond Res. 2016;30(1):93-101.

19. Asano RY, Sales MM, Browne RA, et al. Acute effects of physical exercise in type 2 diabetes: a review. World J Diabetes. 2014;5(5): 659-665.

20. Juffer P, Bakker AD, Klein-Nulend J, Jaspers RT. Mechanical loading by fluid shear stress of myotube glycocalyx stimulates growth factor expression and nitric oxide production. Cell Biochem Biophys. 2014; 69(3):411-419.

21. Santana HA, Moreira SR, Asano RY, et al. Exercise intensity modulates nitric oxide and blood pressure responses in hypertensive older women. Aging Clin Exp Res. 2013;25(1):43-48.

22. Huang AN, Sun D, Koller A, Kaley G. Gender difference in flowinduced dilation and regulation of shear stress: role of estrogen and nitric oxide. Am J Physiol Regul Integr Comp Physiol. 1998;275(5): R1571-R1577.

23. Chambliss KL, Shaul PW. Estrogen modulation of endothelial nitric oxide synthase. Endocr Res. 2002;23(5):665-686.

24. Carpio-Rivera E, Moncada-Jiménez J, Salazar-Rojas W, Solera-Herrera A. Acute effects of exercise on blood pressure: a meta-analytic investigation. Arq Bras Cardiol. 2016;106(5):422-433. 
25. Foster C, Florhaug JA, Franklin J, et al. A new approach to monitoring exercise training. J Strength Cond Res. 2001;15(1):109-115.

26. Rhea MR. Determining the magnitude of treatment effects in strength training research through the use of the effect size. J Strength Cond Res. 2004;18(4):918-920.

27. Day ML, McGuigan MR, Brice G, Foster C. Monitoring exercise intensity during resistance training using the session RPE scale. $J$ Strength Cond Res. 2004;18(2):353-358.

28. Kraemer WJ, Ratamess NA. Fundamentals of resistance training: progression and exercise prescription. Med Sci Sports Exerc. 2004; 36(4):674-688.

29. Uchida MC, Nishida MM, Sampaio RAC, et al. Thera-band® elastic band tension: reference values for physical activity. J Phys Ther Sci. 2016;28(4):1266.

30. Uchida MC, Teixeira LF, Godoi VJ, et al. Does the timing of measurement alter session-RPE in boxers? J Sports Sci Med. 2014;13(1): 59-65.

31. Martins WR, de Oliveira RJ, Carvalho RS, de Oliveira Damasceno V, da Silva VZ, Silva MS. Elastic resistance training to increase muscle strength in elderly: a systematic review with meta-analysis. Arch Gerontol Geriatr. 2013;57(1):8-15.

32. Colado JC, Triplett NT. Effects of a short-term resistance program using elastic bands versus weight machines for sedentary middle-aged women. J Strength Cond Res. 2008;22(5):1441-1448.

33. Sweet TW, Foster C, McGuigan MR, Brice G. Quantitation of resistance training using the session rating of perceived exertion method. J Strength Cond Res. 2004;18:796-802.
34. Chobanian AV, Bakris GL, Black HR, et al; National Heart L, and Blood Institute Joint National Committee on Prevention, Detection, Evaluation, and Treatment of High Blood Pressure; National High Blood Pressure Education Program Coordinating Committee. The Seventh Report of the Joint National Committee on Prevention, Detection, Evaluation, and Treatment of High Blood Pressure: the JNC 7 report. JAMA. 2003;289:2560-2572.

35. Diaz MM, Bocanegra OL, Teixeira RR, Soares SS, Espindola FS. Salivary nitric oxide and alpha-amylase as indexes of training intensity and load. Int J Sports Med. 2013;34:8-13.

36. Rezk CC, Marrache RC, Tinucci T, Mion D Jr, Forjaz CL. Post-resistance exercise hypotension, hemodynamics, and heart rate variability: influence of exercise intensity. Eur J Appl Physiol. 2006;98:105-112.

37. Chowdhary S, Townend JN. Role of nitric oxide in the regulation of cardiovascular autonomic control. Clin Sci (Lond). 1999;97:5-17.

38. Flesch M, Kilter H, Cremers B, et al. Acute effects of nitric oxide and cyclic GMP on human myocardial contractility. J Pharmacol Exp Ther. 1997;281:1340-1349.

39. Balligand JL, Kelly RA, Marsden PA, Smith TW, Michel T. Control of cardiac muscle cell function by an endogenous nitric oxide signaling system. Proc Natl Acad Sci U S A. 1993;90:347-351.

40. Moraes MR, Bacurau RFP, Simões HG, et al. Effect of 12 weeks of resistance exercise on post-exercise hypotension in stage 1 hypertensive individuals. J Hum Hypertens. 2012;26(9):533-539. 


\section{Supplementary materials}

Table SI Behavior of hemodynamic parameters after the experimental sessions in hypertensive patients

\begin{tabular}{|c|c|c|}
\hline Variables & RT & PT \\
\hline \multicolumn{3}{|l|}{ SBP } \\
\hline IA & $1.4 \pm 10.9$ & $-4.6 \pm 7.8$ \\
\hline $5^{\prime \prime}$ & $-1.4 \pm 15.6$ & $-9.0 \pm 6.5$ \\
\hline $10^{\prime \prime}$ & $-6.2 \pm 16.3$ & $-6.8 \pm 10.8$ \\
\hline $15^{\prime \prime}$ & $-1.8 \pm 4.8$ & $-12.8 \pm 6.2^{*}$ \\
\hline $30^{\prime \prime}$ & $-2.0 \pm 4.5$ & $-17.4 \pm 5.9 * * *$ \\
\hline $45^{\prime \prime}$ & $-1.4 \pm 12.6$ & $-12.0 \pm 13.3^{*}$ \\
\hline $60^{\prime \prime}$ & $-5.4 \pm 10.2$ & $-7.4 \pm 10.0$ \\
\hline \multicolumn{3}{|l|}{ DBP } \\
\hline IA & $0.8 \pm 3.1$ & $0.2 \pm 4.9$ \\
\hline $5^{\prime \prime}$ & $-0.8 \pm 5.7$ & $0.8 \pm 9.2$ \\
\hline $10^{\prime \prime}$ & $-2.4 \pm 5.5$ & $-7.8 \pm 9.0$ \\
\hline $15^{\prime \prime}$ & $-3.8 \pm 3.7$ & $-1.8 \pm 5.9$ \\
\hline $30^{\prime \prime}$ & $-0.2 \pm 5.2$ & $-2.2 \pm 9.4$ \\
\hline $45^{\prime \prime}$ & $0.0 \pm 4.4$ & $-3.0 \pm 7.0$ \\
\hline $60^{\prime \prime}$ & $-1.6 \pm 4.0$ & $-4.4 \pm 6.3$ \\
\hline \multicolumn{3}{|l|}{ HR } \\
\hline IA & $-26.8 \pm 32.5 * *$ & $1.6 \pm 6.1$ \\
\hline $5^{\prime \prime}$ & $-27.6 \pm 31.4^{* *}$ & $-2.6 \pm 5.0$ \\
\hline $10^{\prime \prime}$ & $-27.0 \pm 31.2 * *$ & $-2.6 \pm 3.5$ \\
\hline $15^{\prime \prime}$ & $-28.0 \pm 31.4 * *$ & $-4.0 \pm 4.8$ \\
\hline $30^{\prime \prime}$ & $-26.6 \pm 33.1 * *$ & $-5.8 \pm 4.0$ \\
\hline $45^{\prime \prime}$ & $-29.8 \pm 30.9 * *$ & $-6.4 \pm 3.3$ \\
\hline $60^{\prime \prime}$ & $-28.8 \pm 31.6 * *$ & $-17.6 \pm 23.7^{*}$ \\
\hline
\end{tabular}

Notes: $* P<0.05$ vs baseline data prior to exercise; $* * P<0.01$ vs baseline data prior to exercise; $* * * P<0.001$ vs baseline data prior to exercise. Data presented as mean \pm standard deviation.

Abbreviations: RT, resistance training; PT, power training; SBP, systolic blood pressure; IA, immediately after; DBP, diastolic blood pressure; HR, heart rate.
Table S2 ES of hemodynamic parameters after the experimental sessions in hypertensive patients

\begin{tabular}{cll}
\hline Variables & RT & PT \\
\hline SBP & & \\
IA & -0.18 (trivial) & 0.83 (small) \\
$5^{\prime \prime}$ & 0.12 (trivial) & 1.95 (moderate) \\
I0" & 0.53 (small) & 0.89 (small) \\
I5" & 0.53 (small) & 2.91 (moderate) \\
$30^{\prime \prime}$ & 0.62 (small) & 4.17 (moderate) \\
$45^{\prime \prime}$ & 0.76 (small) & 1.27 (moderate) \\
$60^{\prime \prime}$ & 0.74 (small) & 1.04 (small) \\
DBP & & \\
IA & -0.36 (trivial) & -0.05 (trivial) \\
$5^{\prime \prime}$ & 0.19 (trivial) & -0.12 (trivial) \\
I0" & 0.61 (small) & 1.22 (small) \\
I5" & 1.45 (moderate) & 0.43 (trivial) \\
$30^{\prime \prime}$ & 0.05 (trivial) & 0.33 (trivial) \\
$45^{\prime \prime}$ & 0 (trivial) & 0.60 (small) \\
$60^{\prime \prime}$ & 0.56 (small) & 0.98 (small) \\
HR & & \\
IA & 1.16 (small) & -0.37 (trivial) \\
$5^{\prime \prime}$ & 1.24 (small) & 0.73 (small) \\
I0" & 1.22 (small) & 1.05 (small) \\
I5" & 1.26 (moderate) & 1.17 (small) \\
$30^{\prime \prime}$ & 1.13 (small) & 2.05 (moderate) \\
$45^{\prime \prime}$ & 1.36 (moderate) & 2.74 (moderate) \\
$60^{\prime \prime}$ & 1.28 (moderate) & 1.05 (small) \\
\hline
\end{tabular}

Note: Data presented as Cohen's $d$.

Abbreviations: ES, effect size; RT, resistance training; PT, power training; SBP, systolic blood pressure; IA, immediately after; DBP, diastolic blood pressure; HR, heart rate.

Table S3 Comparison of rest and the mean of the moments after exercise in hypertensive patients

\begin{tabular}{lll}
\hline Variables & RT & PT \\
\hline SBP & & \\
Rest & $130.6 \pm 7.5$ & $|4| .0 \pm 16.8$ \\
$\Delta$ I hour & $-3.5 \pm 5 . I$ & $-13.1 \pm 7.5^{*}$ \\
ES & 0.97 (small) & 2.47 (large) \\
Rest & $82.4 \pm 9.5$ & $72.0 \pm 7.0$ \\
DBP & & \\
$\Delta$ I hour & $-1.1 \pm 3.8$ & $-6.7 \pm 4.4^{*}$ \\
ES & 0.40 (trivial) & 2.15 (large) \\
Rest & $95.2 \pm 21 . I$ & $74.6 \pm 10.2$ \\
HR & & \\
$\Delta$ I hour & $-18.3 \pm 10.4^{*}$ & $-1.4 \pm 2.4$ \\
ES & 2.48 (large) & 0.82 (small) \\
\hline
\end{tabular}

Notes: $* P<0.05$ vs baseline data prior to exercise. Data presented as mean \pm standard deviation and Cohen's d (ES).

Abbreviations: RT, resistance training; PT, power training; SBP, systolic blood pressure; ES, effect size; DBP, diastolic blood pressure; HR, heart rate.

\section{Dovepress}

\section{Publish your work in this journal}

Clinical Interventions in Aging is an international, peer-reviewed journal focusing on evidence-based reports on the value or lack thereof of treatments intended to prevent or delay the onset of maladaptive correlates of aging in human beings. This journal is indexed on PubMed Central, MedLine,
CAS, Scopus and the Elsevier Bibliographic databases. The manuscript management system is completely online and includes a very quick and fair peer-review system, which is all easy to use. Visit http://www.dovepress. com/testimonials.php to read real quotes from published authors. 